\title{
The Model of the Thin Metal Layer Electrical Conductivity in the Case of Different Reflection Coefficients of its Surfaces
}

\author{
Utkin A.I., Yushkanov A.A. \\ Moscow State Regional University, 10a, Radio st., 105005, Moscow, Russia \\ *Corresponding Author: utkin.a.i@mail.ru
}

Copyright (C) 2013 Horizon Research Publishing All rights reserved.

\begin{abstract}
Local electrical conductivity of a thin metal film, with the different coefficients of the reflection surfaces, is calculated in this article. The dependence of conductive function on the dimensionless frequency of volume electrons collisions, the dimensionless frequency of the external field and the dimensionless distance to the upper surface layer is analyzed. The case of thin film, when the thickness a lot more than average free path length of electrons, which led to the classic results for the electrical conductivity, is considered. The kinetic equation of Boltzmann in approximation of electrons relaxation time is used.
\end{abstract}

Keywords Thin Metal Layer, The Reflectivity Coefficient, Local Electrical Conductivity, Length Free Path Of Electrons, The Dimensionless Frequency of Volume Electrons Collisions, The Dimensionless Frequency of the External Field, The Dimensionless Distance to the Upper Surface Layer

\section{Introduction}

Currently microelectronics, optoelectronics and thin-film technologies are actively developing. As this is an important application thin films, including polycrystalline and metal films $[1,2,3,4]$. Judicious use of thin films in micro - and optoelectronics allows them to go to a qualitatively new level of development. For example, thin films can be used as conductive, reflective and protective coatings. Therefore, must be high requirements are needed to the quality, parameters and properties $[5,6]$ of thin films.

At this stage development of thin-film technologies is the greatest interest in the investigation of thin films electromagnetic properties at various ranges of frequencies (for example, in [7] considered the interaction of H-wave with a thin metal film).

In this article, it seems logical to investigate the thin metallic film local electrical conductivity, when its thickness is comparable with the average of the mean free path of electrons. Quantum effects in this case are ignored. Accounting for the effects was considered [8], in the case of quantum film in a dielectric environment.

It is known that the electrical conductivity of thin metal films can be much less than the electrical conductivity of metals in a massive form. A similar phenomenon was found in 1857 year by M. Faraday. Later, this phenomenon was theoretically described by K. Fuchs, and then analyzed by E. Sondheimer [9] in view the scattering of electrons by surfaces of thin metal layer. Later K. Fuchs [10] was able to get the analytical formula for the local electrical conductivity of a thin metal layer. However, it was not considered that reflection coefficients of thin metal layer surfaces can be different. Also not considered the frequency dependence of the electrical conductivity. Do accounting of the foregoing. The aim of this article is to the Local electrical conductivity.

\section{Problem Definition and Methods}

Consider the thin conductive layer thickness of $a$, with reflection coefficients top $\left(q_{1}\right)$ and lower $\left(q_{2}\right)$ surfaces layer, in the case of the homogeneous periodic time electric field $\mathbf{E}$. The electric field is parallel the conductive layer and it is directed along the $X$-axis, while $Z$-axis is directed into the layer. Homogeneous periodic time electric field varies according to the equation of electromagnetic wave propagation:

$$
\mathbf{E}=\mathbf{E}_{0} \exp (-i \omega t)
$$

$\omega$ - the frequency of the alternating voltage applied to the conducting layer.

Skin effect is not taken into account. Accounting skin effect was considered [11] in the case of a thin cylindrical wire.

Kinetic equation of Boltzmann in the relaxation time approximation of the electrons (in the Tau approximation) [12] was used for obtaining formula of the local electrical conductivity: 


$$
\begin{array}{r}
\frac{\partial f}{\partial t}+v_{z} \frac{\partial f}{\partial z}+\frac{e E}{m} \frac{\partial f_{0}}{\partial v_{x}}=-\frac{f-f_{0}}{\tau}, \\
f_{0}(\varepsilon)=\frac{1}{\exp ((\varepsilon-\mu) / k T)+1}
\end{array}
$$

$f_{0}(\varepsilon), f, e, m, v_{z}, v_{x}, \tau$ и $\mu$ - are respectively, the function of Fermi - Dirac, distribution function of the electrons in the presence of an external electric field, electron charge, effective mass of electron, the projection of conduction electrons velocity, electronic relaxation time and the chemical potential.

For the functions $f_{0}(\varepsilon)$ the approximation is used:

$$
f_{0}=\Theta\left(\varepsilon_{\mathrm{F}}-\varepsilon\right)=\left\{\begin{array}{l}
1,0 \leq \varepsilon \leq \varepsilon_{\mathrm{F}} . \\
0, \varepsilon_{\mathrm{F}}<\varepsilon
\end{array} .\right.
$$

$\varepsilon_{F}=\frac{m v_{F}^{2}}{2}-$ Fermi energy, $\mathrm{vF}-$ speed Fermi.

The value of free electron path $\Lambda$ and electronic relaxation time $\tau$ are related by the formula: $\Lambda=v_{F} \tau$.

Also for the energy $\varepsilon$ and $f_{0}(\varepsilon)$ we have:

$$
\begin{aligned}
& \varepsilon=\frac{m v^{2}}{2}, \\
& f_{0}=\eta\left(\varepsilon_{F}-\varepsilon\right), \\
& \eta(x)=\left\{\begin{array}{l}
1, x>0, \\
0, x<0 ;
\end{array}\right. \\
& \frac{\partial f_{0}}{\partial v_{x}}=\frac{\partial f_{0}}{\partial \varepsilon} \frac{\partial \varepsilon}{\partial v_{x}}=-\delta\left(\varepsilon_{F}-\varepsilon\right) m v_{x}
\end{aligned}
$$

$\delta$ - Delta-function Dirac.

In the linear approximation on the field $\mathbf{E}$ function $f$ can be written as:

$$
f(z, \mathbf{v})=f_{0}(\varepsilon)+f_{1}(z, \mathbf{v}) .
$$

$f_{1}(z, \mathbf{v})$ - small deviation from $f_{0}(\varepsilon) ; f(z, \mathbf{v}) \quad-$ distribution function of the electron velocity in the case of an external electric field.

Then the Boltzmann equation in the Tau approximation would be:

$$
v_{\mathrm{z}} \frac{\partial f_{1}}{\partial \mathrm{z}}+\frac{e E}{m} \frac{\partial f_{0}}{\partial v_{x}}-i \omega f_{1}=-\frac{f_{1}}{\tau}
$$

Solving equation (2) taking into account the boundary conditions for thin conductive layer:

$$
f_{1}\left(v_{z}, z=\mathrm{a}\right)=q_{1} f_{1}\left(-v_{z}, z=\mathrm{a}\right), v_{z}>0,
$$

$$
f_{1}\left(v_{\mathrm{z}}, z=0\right)=q_{2} f_{1}\left(-v_{z}, \mathrm{z}=0\right), v_{z}<0 .
$$

$q_{1}$ and $q_{2}$, accordingly, are the reflection coefficients of the upper and lower surfaces layer

Solving the equation (2) and making the necessary transformations, we obtained the analytical formula for the local electrical conductivity of the layer as a function of dimensionless complex frequency scattering of electrons $\Omega$ with reflection coefficients top $\left(q_{1}\right)$ and lower $\left(q_{2}\right)$ surfaces layer:

$$
\sigma=\frac{3 n e^{2} a}{4 m v_{F} \Omega} \int_{0}^{\frac{\pi}{2}} \sin ^{3} \theta \times
$$

$$
\begin{gathered}
\times\left[2+\frac{q_{1}\left(1-\exp (-\Omega / \cos \theta)+q_{2} \exp (-\Omega / \cos \theta)\right)-1}{1-q_{1} q_{2} \exp (-2 \Omega / \cos \theta)} \times \exp (-\Omega \xi / \cos \theta)+\right. \\
+\frac{q_{2}\left(1-\exp (-\Omega / \cos \theta)+q_{1} \exp (-\Omega / \cos \theta)\right)-1}{1-q_{1} q_{2} \exp (-2 \Omega / \cos \theta)} \times \\
\times \exp (-\Omega(1-\xi) / \cos \theta)] d \theta,
\end{gathered}
$$

$\xi=\frac{z}{a}-$ the dimensionless distance to the upper surface layer, $\Omega=\frac{a}{v_{z}}\left(\frac{1}{\tau}-i \omega\right)=x-i y ; \mathrm{n}-$ concentration of conduction electrons.

Simplified formula (3) for electric conductivity finally we got:

$$
\sigma_{a}=\sigma_{0} \lambda a \int_{0}^{1}\left(1-t^{2}\right) \times
$$

$$
\begin{aligned}
& \times\left[2+\left[\frac{q_{1}\left(1-\exp (-(x-i y) / t)+q_{2} \exp (-(x-i y) / t)\right)-1}{1-q_{1} q_{2} \exp (-2(x-i y) / t)} \times\right.\right. \\
& \times \exp (-(x-i y) \xi / t)+
\end{aligned}
$$

$$
+\frac{q_{2}\left(1-\exp (-(x-i y) / t)+q_{1} \exp (-(x-i y) / t)\right)-1}{1-q_{1} q_{2} \exp (-2(x-i y) / t)} \times
$$

$$
\times \exp (-(x-i y)(1-\xi) / t)] d t
$$

$\sigma_{0}=\frac{n e^{2}}{m} \tau \quad, \quad \lambda=\frac{1}{1-i \omega \tau}=\frac{x}{x-i y} \quad ; \quad x=\frac{a}{v_{F} \tau} \quad-\quad$ the dimensionless frequency of bulk electron collisions, $y=\frac{a}{v_{F}} \omega-$ the dimensionless frequency of the electric field. 
Select $\Sigma$ function for further its researching:

$$
\begin{gathered}
\Sigma=\lambda \int_{0}^{1}\left(1-t^{2}\right) \times \\
\times\left[2+\left[\frac{q_{1}\left(1-\exp (-(x-i y) / t)+q_{2} \exp (-(x-i y) / t)\right)-1}{1-q_{1} q_{2} \exp (-2(x-i y) / t)} \times\right.\right. \\
\times \exp (-(x-i y) \xi / t)+ \\
+\frac{q_{2}\left(1-\exp (-(x-i y) / t)+q_{1} \exp (-(x-i y) / t)\right)-1}{1-q_{1} q_{2} \exp (-2(x-i y) / t)} \times \\
\times \exp (-(x-i y)(1-\xi) / t)] d t, \\
\sigma_{a}=\sigma_{0} a \Sigma .
\end{gathered}
$$

Analyze formula (4), and consider the case of thick film, when the thickness a lot more, then free path length of electrons $\Lambda$.

Then the formula (4), in the case of thick film would be:

$$
\sigma_{a}=a \sigma_{0} \frac{1}{1-i \omega \tau}
$$

Formula (5) is Drude formula.

Thus, we have a classical result for conductivity thick film.

\section{Results and Discussion}

Let us consider the dependence of the real and imaginary part of $\Sigma$ function on the dimensionless distance to the upper surface layer $\xi$, the dimensionless frequency of bulk electron collisions $x$, and on dimensionless frequency of the external electric field $y$. For clarity, in the static case $(y=0)$ conductivity value is real.

\section{Conclusions}

After analyzing the graphs it was found that the greatest divergence of curves 1 and 2 in figures 1,3 and 4 is achieved at minimum numerical value of the formula:

$$
1-\left|q_{1}-q_{2}\right|
$$

In figure 2 we can see that the rate of curve increment increases gradually, going from diffuse case $(q=0)$ to the reflection case $(q=1)$

Figure 5 reveals the curious dependence of the real part of $\Sigma$ functions on the dimensionless frequency of bulk electron collisions $x$, with variations of the dimensionless distance to the upper layer surface $\xi$ : smooth decrease of the dimensionless distance to the upper surface layer $\xi$, the rate of increase of the curve increases, and then begins to decrease.

In figure 6 we can see agreement between the experimental data and with our model

It is proved, that we obtained a classical result for conductivity thick film when its thickness $a$ is much greater than the length of the free path of electrons $A$

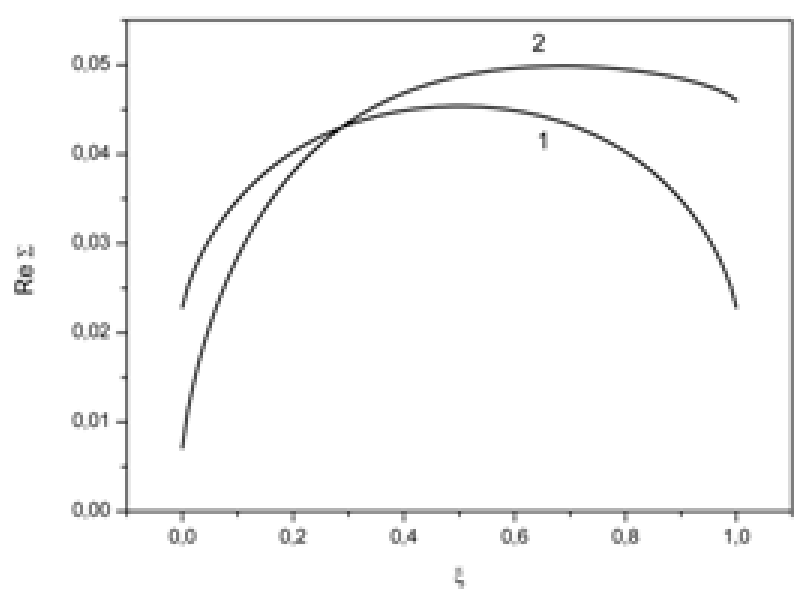

Figure 1. The dependence of the real part $\Sigma$ function on the dimensionless distance to the upper surface layer $\xi$. Curve $1: q_{1}=q_{2}=0.5, x=0.1, y=1$; curve 2: $q_{1}=0.9, q_{2}=0.1, x=0.1, y=1$.

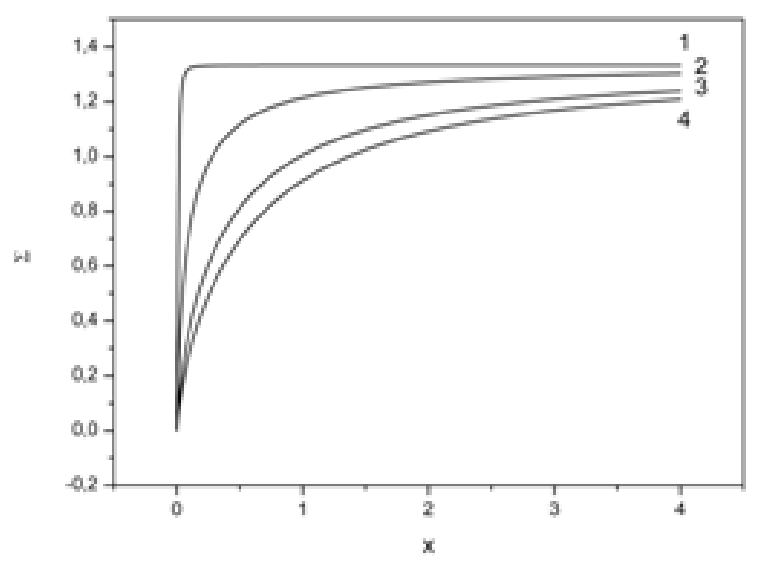

Figure 2. The dependence of the $\Sigma$ function on the dimensionless frequency of bulk electron collisions $x$ (for all curves $y=0$ ). Curve 1: $q_{1}=q_{2}=1$; curve 2: $q_{l}=0.7, q_{2}=0.8$; curve $3: q_{1}=0.2, q_{2}=0.3$; curve $4: q_{1}=q_{2}=0$.

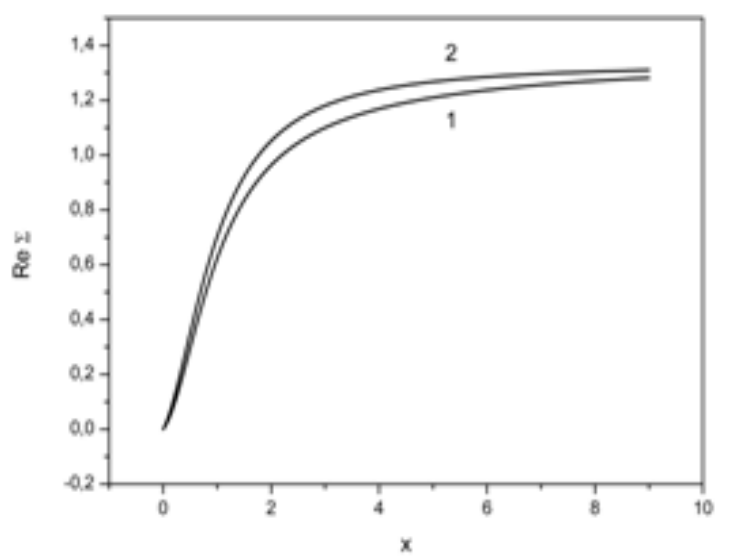

Figure 3. The dependence of the real part $\Sigma$ function on the dimensionless frequency of bulk electron collisions $x$. Curve 1: $q_{1}=q_{2}=0.5, \xi=0.9, y=1$; curve 2 : $q_{1}=0.9, q_{2}=0.1, \quad \xi=0.9, y=1$. 


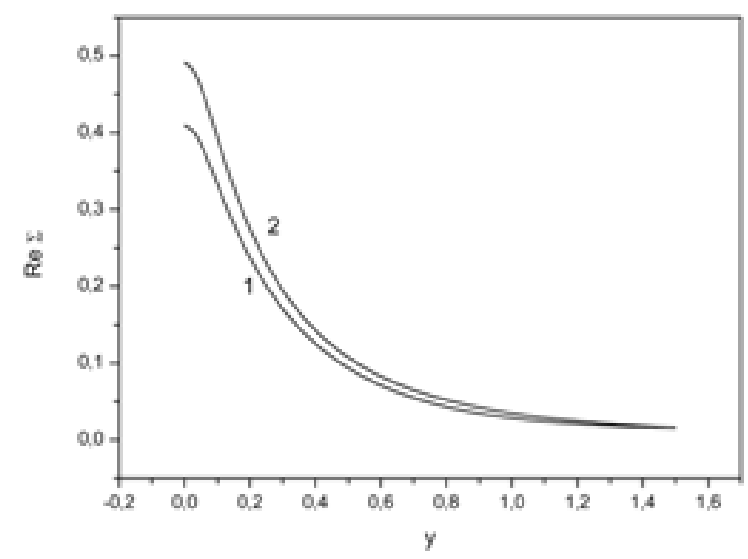

Figure 4. The dependence of the real part $\Sigma$ function on the dimensionless frequency of the external field $y$. Curve 1: $q_{I}=q_{2}=0.5, \quad \xi=0.9, x=0.1$; curve 2: $q_{1}=0.9, q_{2}=0.1, \xi=0.9, \mathrm{x}=0.1$.

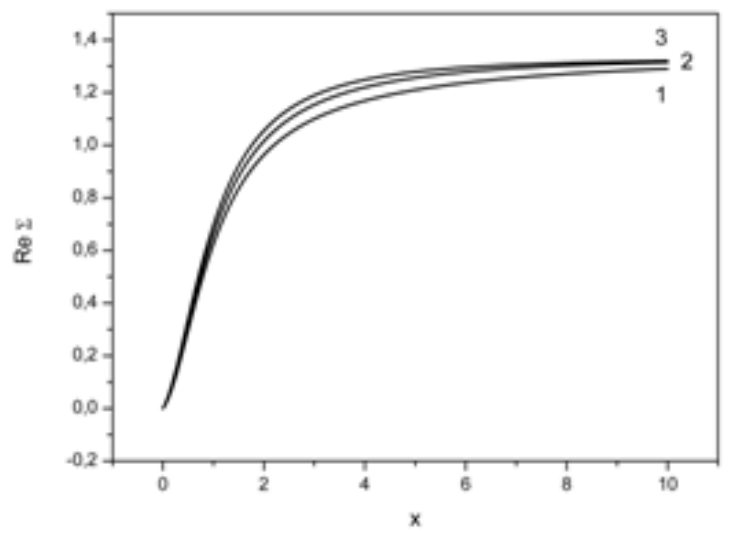

Figure 5. The dependence of the real part $\Sigma$ function on the dimensionless frequency of bulk electron collisions $x$. Curve 1: $q_{l}=q_{2}=0.5, \quad \xi=0.9, y=1$; curve $2: q_{1}=q_{2}=0.5, \quad \xi=0.2, y=1$; curve $3: q_{1}=q_{2}=0.5, \quad \xi=0.5, y=1$.

Make the comparison of some obtained results with the experimental data of [13] article. The numerical value of the average free length of the electrons in comparison with the experiment: $\Lambda=126 \mathrm{~nm}$.

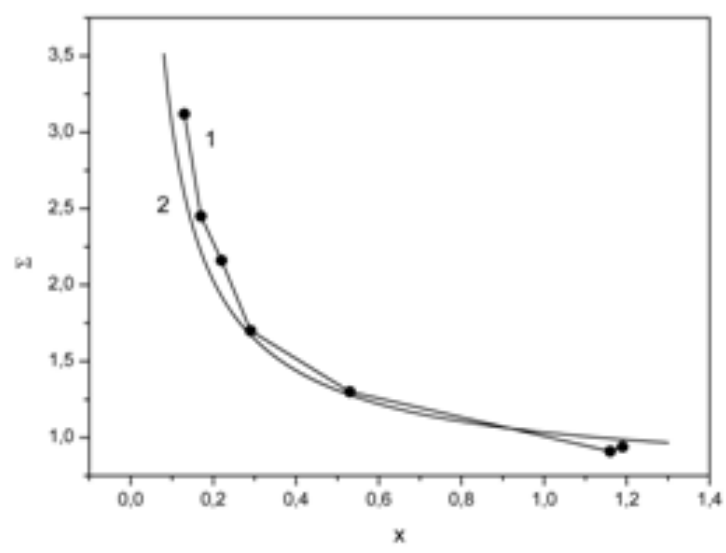

Figure 6. The dependence of the $\Sigma$ function on the dimensionless frequency of bulk electron collisions $x$. Curve 1 is the experimental data, curve 2 is the our model data: $q_{I}=0.1, q_{2}=0.2, y=0$.

\section{REFERENCES}

[1] V.V. Kaminsky, N.N. Stepanov, M.M. Kazanin, A.A. Molodyh, S.M. Soloviev. Electrical conductivity and zonal structure of thin polycrystalline films EuS, Physics of the Solid State, Vol.55, No.5, 991-994, (2013).

[2] H.P. Mayssel, R. Glenk. Technology of thin films, Mir, USSR, (1977).

[3] R. Suhu. Magnetic thin films, Mir, USSR, (1967).

[4] G. Hass. Physics of thin films, Mir, USSR, (1967).

[5] F. Abeles. Optical properties of metal films. Physics of thin films, Mir, USSR, (1973).

[6] K.L. Chopra. Electrical phenomena in thin films, Mir, USSR, (1972).

[7] A.V. Latyshev, A.A. Yushkanov. Interaction of electromagnetic $\mathrm{H}$-waves with the thin metal film, Microelectronics, Vol. 41, No.1, 30-35, (2012).

[8] A.V. Babich, V.V. Pogosov. Quantum metal film in the dielectric environment, Physics of the Solid State, Vol. 55, No.1, 177-185, (2013).

[9] E.H. Sondheimer. The mean free path of electrons in metals, Advances in Physics, Vol.50, No.6, 499-537, (2001).

[10] K. Fuchs. The conductivity of thin metallic films according to the electron theory of metals, Proc. Camb. Phil. Soc, Vol.34, 100-108, (1938).

[11] E.V. Zavitaev, O.V. Rusakov, A.A. Yushkanov. Skin effect in the thin cylindrical wire made of metal, Physics of the Solid State, Vol.54, No.6, 1041-1047, (2012).

[12] A.A. Abrikosov. Bases of the metals theory: a Training manual, Nauka, USSR, (1987).

[13] Sun Tik, Yao Bo, Warren Andrew P., Kumar Vineet, Roberts Scott, Barmak Katayun, Coffey Kevin R. Classical size effect in oxide-encapsulated $\mathrm{Cu}$ thin films: impact of grain boundaries versus surfaces on resistivity, J. Vac. Sci. Technol. A, Vol.26, No.4, 605-609, (2008). 